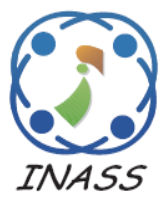

\title{
Employing MZI-SOA to Design and Simulate the Optical OR Gate at A High Bit Rates of 250 Gbps
}

\author{
Ahmed Aldhahab $^{1 *} \quad$ Ibrahim Murdas ${ }^{1}$ \\ ${ }^{1}$ Department of Electrical Engineering, University of Babylon, Babylon, Iraq \\ * Corresponding author's Email: aldhahab2012@knights.ucf.edu
}

\begin{abstract}
Conventional logic gates failed to process data at a high bit rate. Therefore, researchers began to study the design of these gates with optical technology, which is characterized by high bit rate data processing. The non-linear behavior is a drawback in semiconductor optical amplifier (SOA). This nonlinearity property of the SOA is used in this paper to implement the OR optical gates. Mach-Zander interferometer (MZI)-based structures are among the numerous of structures that are available to transform a modulation of phase into a modulation of intensity. These are widely used by electro-optic (EO), or thermo-optic (TO) impacts to create various applications like optical modulators, splitters, switches, etc. In this paper, the MZI is used as switches and the SOA is employed with cross-gain modulation (XGM) to implement the optical logic gate OR at a high bit rate of $250 \mathrm{Gbps}$. Several experimental results were performed on the proposed design of the OR gate until the optimum value of the injection current, which is $0.08 \mathrm{~A}$, was obtained. This optimum value led to achieve the lowest BER, highest Quality factor, and lowest power consumption, which are $0,60.7989$, and $31.13 \times 10-3$, respectively. The experimental results of the proposed design outperformed those results obtained by other state-of-the-art designs.
\end{abstract}

Keywords: Optical semiconductor device, Optical kerr effect, Cross gain modulation, Optical OR gate.

\section{Introduction}

The demand to use the electronic devices with high specifications in data processing as well as data transfer at a high bit rate is increased in the recent years. Therefore, all optical logic gates have recently received a great deal of interest in various fields, the most important one is the optical networks field, as it enables the performance of many advanced functions and relied on achieving them as optical logic functions on the non-linear effects found in semiconductors and optical fibers. The main reason for developing the optical networks is the discovery of optical amplifiers. Optical amplifiers can be divided into two parts. The first part is the optical fiber amplifiers while the second part is the semiconductor optical amplifiers (SOA). The optical gates are used in high-speed communication systems as well as processing optical signals, such as coding, decoding, decision-making, etc. [1-3].
All optical logic switches (gates) are employed by either linear or nonlinear designs. The nonlinear effect of the single channel is accomplished by selfphase modulation (SPM) in which each signal propagates in a channel modulate its own phase. The nonlinearity effect of the wavelength division multiplexing (WDM) is classified by XGM, XPM, and FWM. The wavelength conversion is accomplished by the nonlinearity effect in XGM. Also, XGM nonlinear effect is used to achieve the gain saturation. In XPH, the phase of each channel is modified by the power of co-propagation channels. Finally, in FWM, it is required into more than one channel to mixed together to transfer the data energy to the new ones $[4,5]$.

There were many algorithms proposed to implement the optical logic gates. In 2014, Ankur Saharia, et al. [6] have demonstrated all optical NAND gate by exploiting XGM behavior of SOA. Output signal versus input signal was investigated and verified through their simulation. The proposed 
system was evaluated at $10 \mathrm{~Gb} / \mathrm{s}$. The authors in [7] used semiconductor optical amplifier (SOA) in conjunction with the Michelson interferometer (MI) to implement the AND optical logic gate. In [7], the SOA is implemented using cross-gain modulation (XGM) technique and the bit rate was $10 \mathrm{~Gb} / \mathrm{s}$. M. Islam and M Barsha in 2018 [8] showed that the MZI could be used as a switch when it is connected either on cross or bar state. They demonstrated that when MZI used as a switch, all optical logic gate networks will be implemented easily. Both XOR and NAND optical logic gates were proposed in [6]. In [6], the authors used gold disk-shaped nanoparticles to implements the logic gates. The structure of the proposed system contained a non-periodic array of disk-shaped nanoparticles. Then, they demonstrated that the principal operation of the optical logic gates, XOR and NAND, is based on the destructive and constructive interference between the input signals. EL-Saeed, et. al in 2016 [9] extended their previous work with new design that was based on SOA-MZI to design all optical logic gates. In [9], the new design can deal with high number of bits that reach to 16 bits. The new design of the XOR logic gates can operate as a NOR gate and multi-function as an OR gate at various wavelengths. The last gate designed in [9] was XNOR that implemented by using MachZehnder Modulator (MZM). The authors in [10] proposed a system that combined all optical logic gates in single structure through connecting multiple SOA in a cascade. The performance analysis of each optical logic gate was obtained by varying the functional parameters of the SOA's active region. The four-waves mixing (FWM) and cross-gain modulation (XGM) were considered in [10] to obtain all optical logic gates. A. H. Neama and I. S. Desher [22] in 2017 proposed an optical circuit design that was based on SOA and MZI to perform the optical logic gate OR and NOT. The authors simulated both OR and NOT gate at different but rates of $10 \mathrm{Gbps}$, $20 \mathrm{Gbps}$, and $40 \mathrm{Gbps}$ The maximum Quality factors (Q-factor) achieved for the OR and the NOT logic gate designs were 5.482 and 41.0243, respectively, for a bit rate of $10 \mathrm{Gbps}$. The multifunctional optical logic gate circuits, which are AND, NOR and exclusive-NOR (XNOR) were proposed in [23]. The authors in [23] used return to zero format in SOA to implement the multifunctional optical gates. The Qfactor obtained using the proposed design was 16.65 , 12.05 , and 10.13 and the bit error rate was less than $10^{-9}$ for all circuits.

In this paper, the SOA is employed with crossgain modulation (XGM) to implement the optical logic gate OR at a high bit rate, which is 250 Gbps. The nonlinearity property of the SOA is used in this paper to simulate the optical logic gate (OR). The MZI is employed in this paper as a switch. SOA is injected with different values of the injunction currents and the optimum value, $0.08 \mathrm{~A}$, is chosen to accomplish the highest Q-factor and lowest BER, which are 60.7989 and 0 , respectively. The experimental results, shown in Table 2, in terms of BER and Q-factor obtained by the design proposed are outperformed those results obtained by [22, 23]. The highest Q-factors obtained by [22, 23] was 27.325 and 37.61 , respectively. These results were achieved due to the system parameters chosen to design the optical logic gates in both [22, 23].

The rest of the paper is arranged as: In section 2, some backgrounds on SOA are presented. The principle of operation of the optical OR gate is explained in section 3. Simulation of the optical OR gate is presented in section 4. In section 5, the simulation results are illustrated. Finally, the conclusion is discussed in section 5 .

\section{Background}

\subsection{Semiconductor optical amplifier (SOA)}

Three types of optical amplifiers are commonly used in many applications to amplify the optical signal. The first one is called an erbium-doped fiber amplifier ((EDFA)). The second one is attributed to the Raman amplifier. The last one is a semiconductor optical amplifier (SOA). Recently, SOA received great deal of attention in the recent years. It is widely used by a researcher since SOA has some advantages, such as low-power consumption, short period of latency, high nonlinearity, stability, and compactness. It can be used to amplify the signal with different wavelengths. This amplifier is coated with an insulating material from the inside in order to prevent the process of reflection that causes feeding. This type differs from other types of amplifiers in a way that it can achieve reflection electrons or holes instead of ions in Different Energy levels [11, 12].

SOA is one of the most important devices in optical communications, which is commonly used in the process of developing communication systems. SOA applications are including signal amplification, wavelength conversion, embedded amplification, and many other important applications. Usually, SOA does not necessary require any process to convert the signal from optical to electrical and vice versa. This is the most prominent features of the SOA. All types of SOA device have the same size, low energy consumption, and high speed for processing information. There are two types of SOA. The first one is the Fabry-Perot SOA (FP-SOA) in which the 


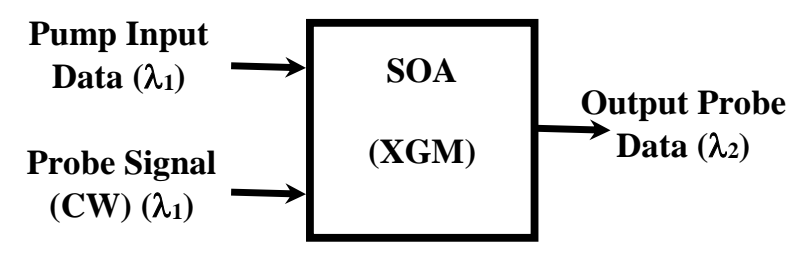

Figure. 1 XGM

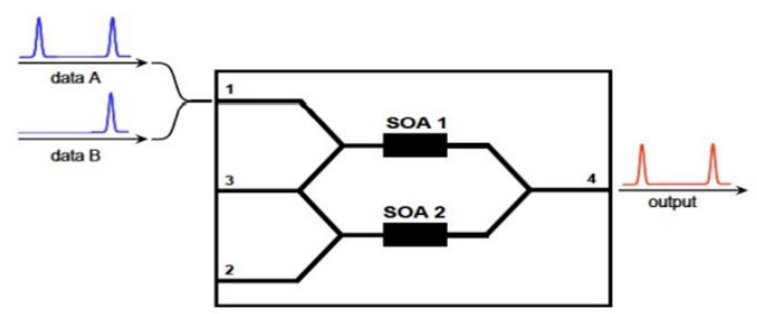

Figure. 2 The OR logic gate principal process utilizing SOA-MZI

end facets repercussions are respectable, and the second one is the travelling-wave SOA (TW-SOA). SOAs with facet reflectivity can be obtained by using anti-reflection coatings [12-14].

\subsection{SOA non-linearity}

The design circuit of the three non-linear processes, which are cross-gain modulation, Cross phase modulation (XPM), and Four-wave mixing (FWM), consists of at least one SOA that is connected with other types of optical devices, such as Mach-Zehnder interferometers (MZI), optical fiber, block of optical gain, etc. in order to implement all optical logic gates. The structure of the SOA in conjunction with the optical fiber is limited to the high-speed because of the phase recovery characteristics and the slower gain. Therefore, the MZI is the right choice to overcome the limitation of the high bit rates $[15,16]$.

The results of the research in high-speed optical communication systems are led to use the wavelength division multiplexing (WDM) technology as the impact of nonlinearity. There are two main groups of fibrous nonlinearities related to either the Kerr optical effect or the stimulated scattering.

\subsection{Optical kerr effect}

The Kerr effect is the simplest and the most common non-linear effect in optical fiber. When the optical intensity increases, the phase delay in the fiber gets larger. It depends on the changes of the refractive index, and it is proportional to the power of the signal $\mathrm{P}(\mathrm{t})$. Hence the change in the refractive index is proportional to the square of the intensity of the light.
The refractive index is denoted by the following equation [17] :

$$
n(w, P(t))=n_{0}(\omega)+n_{2} \frac{P(t)}{A_{e f f}}
$$

where $n_{0}$ is the linear part of the refractive index, $n_{2}$ is the nonlinear part of the refractive index and $A_{\text {eff }}$ is the effective area of the optical fiber.

The non-linearity coefficient $(\boldsymbol{\gamma})$ combines the nonlinear refractive index and the effective core area of the fiber as shown in Eq. (2) $[18,19]$ :

$$
\gamma=\frac{2 \pi}{\lambda} \frac{n_{2}}{A_{\text {eff }}}
$$

where $\lambda$ is the wavelength of the applied light. Although the refractive index is a very weak function based on a signal power, the power of the optical amplifiers and transmission distances make it no longer negligible in modern optical communication systems. Due to the Kerr effect in optical fiber, various nonlinear effects, namely, Self-Phase Modulation (SPM), Cross-Phase Modulation (XPM), and Four-Wave Mixing (FWM) and nonlinear selffocusing $[17,19]$.

\subsection{Cross gain modulation (XGM)}

The basic principle of the cross-gain modulation (XGM) is based on modulating the gain in the SOA through the gain saturation effect. Therefore, the nonlinear process of XGM is based on the saturation of the gain. XGM is employed in the wavelength conversion especially when the strong input power signal is utilized to saturate the gain of the OSA, hence modulates the continuous waveguide signal (CW) to produce new wavelength. As soon as the strong input signal is applied, the refractive index will change in the medium because of the third-order of the non-linearity effect in the SOA. The XGM is suffer from the slower recovery time within SOA. Compared to XPM-SOA and FWM-SOA wavelength conversion, XGM-SOA is considered the simplest wavelength conversion. The gain will be lesser when the input signal is low in comparison with the gain obtained when the input signal is high due to the free carrier resulted from the high input signal. The XGM is shown in Fig. 1. There are some disadvantages of using XGM, which are polarity inversion of the output data compared to the input ones, poor extinction ratio, high optical input power required to achieve the gain saturation in SOA $[20,21]$. 


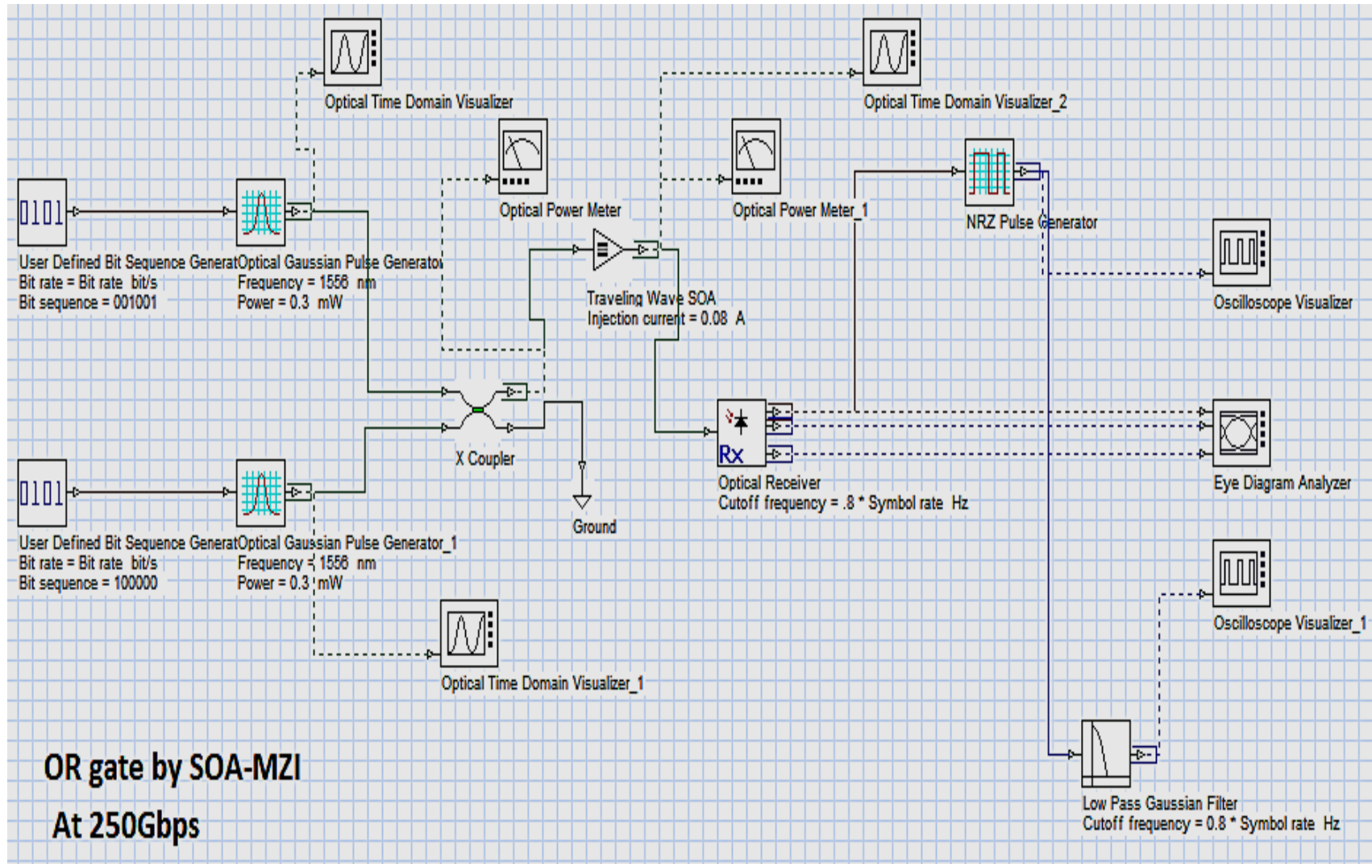

Figure. 3 The circuit diagram for the optical OR gate

\section{Principle operation of the optical OR gate}

To implement the optical OR gate, the principle of mutual gain modulation or the so-called XGM is employed based on the SOA_MZI combination. The principle of the OR gate is explained in the Fig. 2.

The optical OR gate principle of operation depends on the saturation of the gain in the semiconductor optical amplifier. Two streams of data are combined and coupled at certain wavelengths and input to the gain spectrum of the semiconductor optical amplifier. The output of the optical logic gate OR is achieved by the SOA output. The output power $P$ out is larger than $P$ input since the $P$ out is saturated by the input powers. When both data signal A and B shown in Fig. 4 are logic "1", then the output will be corresponding to logic "1" too. However, it is important to note that the input pulsed power must be accurately regulated based on the saturation point of the SOA and the wavelength must be within the gain spectrum of the SOA.

At the input port 1, the information signals are combined and transmitted through the upper input port of the SOAs, in this status, the upper input of the SOA is one. At the output port 4, the signal amplification has to be accomplished by a $3 \mathrm{~dB}$ coupler between the input signals to satisfy the OR gate. In this case, since the inputs and the output signal must be at similar wavelength, there is no need to employ the CW signal for executing process. Hence, there is no need to wavelength conversion.

\section{Simulation of the OR optical gate}

The optical OR gate is implemented by relying on the SOA-MZI and by using Opti-Wave software 17. Fig. 3 illustrates the circuit diagram of the optical OR gate. The components that are used in the designing the optical OR gate are:

a) General Optical Gaussian Pulse Generator: It generates a series of optical Gaussian pulse.

b) Optical Time Domain Visualizer: It displays the modulated optical signal in time domain.

c) Optical Power Meter: It measures the power of the optical signal passed through the optical cable.

d) X-Coupler: It is used for either combing or splitting the optical signal.

e) SOA: Semiconductor Optical Amplifier.

f) Optical Receiver: It is used to convert the optical signal into electrical signal.

g) NRZ Pulse Generator: it is used to generate the sequence of the non-return to zero pulses, which are coded by a digital signal. 
Data $A=001001$

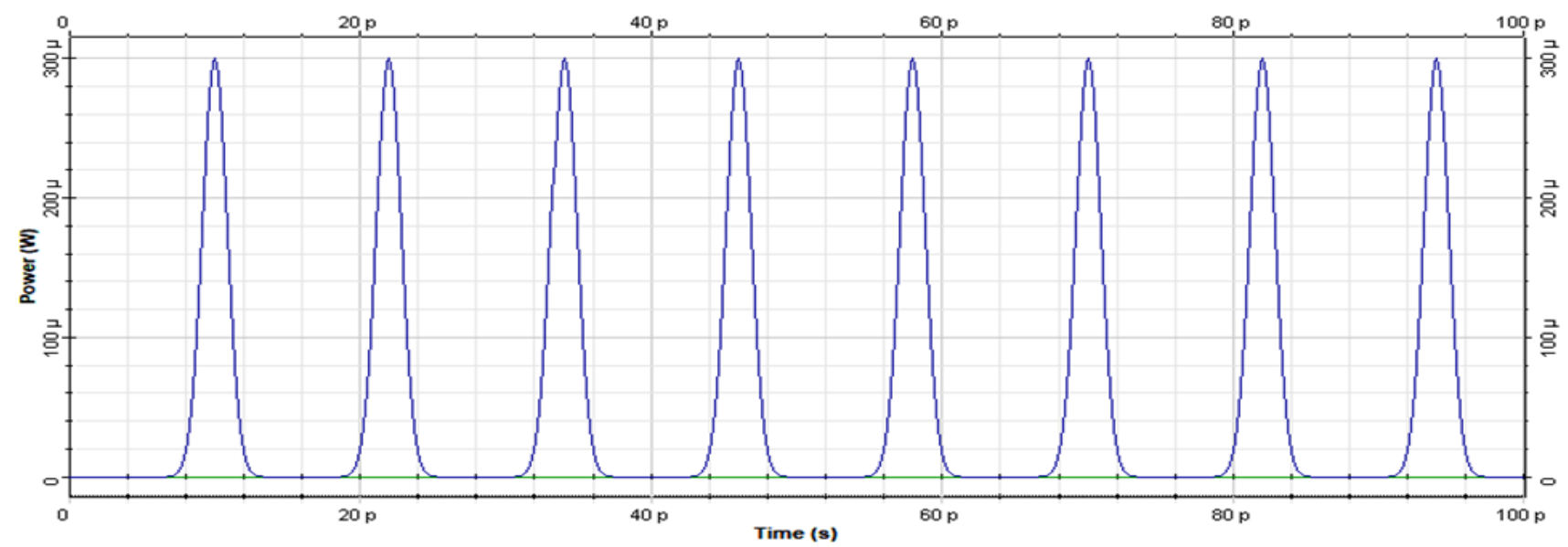

(a)

Data $\mathrm{B}=\mathbf{1 0 0 0 0 0}$

Optical Time Domain Visualizer_1

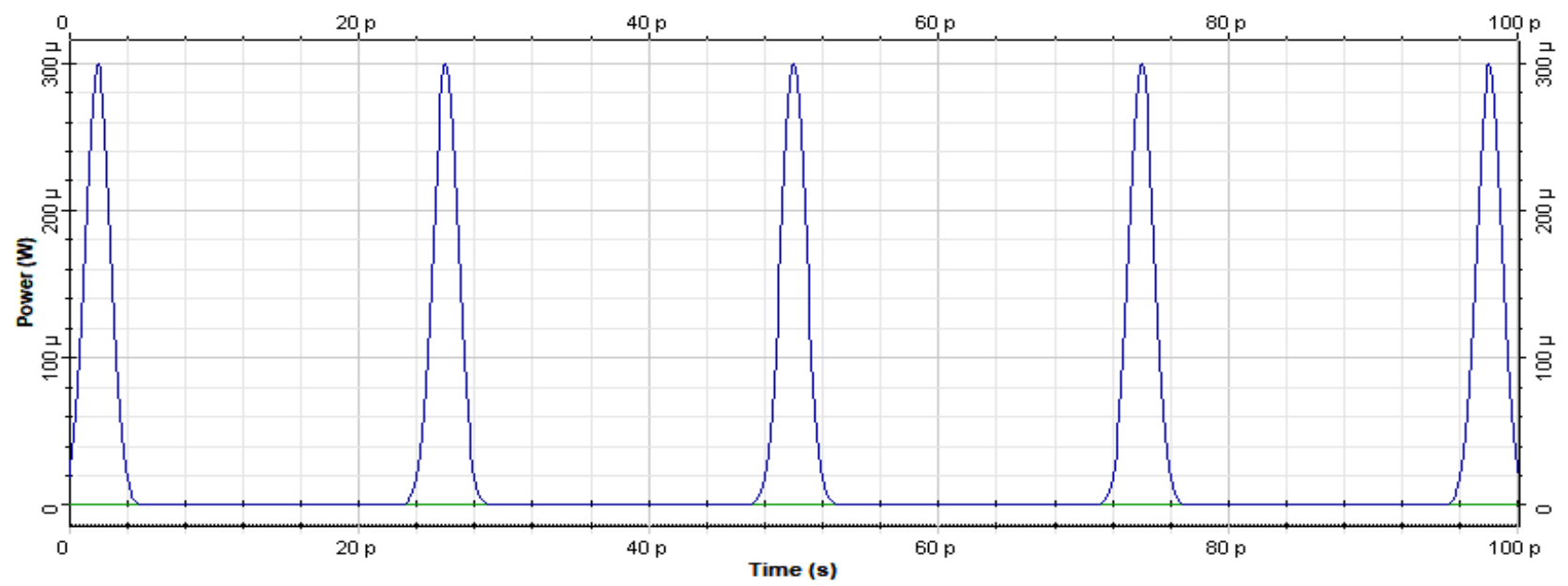

(b)

Figure. 4 The input data waveform to the optical logic gate OR: (a) shows data A, (b) shows

h) Eye Diagram Analyzer: It determine the quality of the received signal. It can measure various metrics, such as BER, eye-opening, Q-factor, eye-closure, etc.

i) Low-Pass Gaussian Filter: It is a filter that has impulse response look like a Gaussian function.

j) Oscilloscope Visualizer: It displays the electrical signal in time domain.

\section{The simulation results}

In this paper, the simulation results of the optical OR gate, shown in Fig. 5, is presented and discussed in this section. After testing the SOA using different values of injunction current, the optimum current value determined to implement the optical logic gate $\mathrm{OR}$ at a high bit rate is obtained. These optimum values of the injunction currents achieve the lowest value of the BER and the largest value of the Quality factor (Q). After settling the right value of the injection current, the optical logic gate OR gate can be simulated by the high bit rate. In this paper, the design of the OR optical logic gate is tested at 250 Gbps bit rate. In this implementation, input and output power, Q factor, and BER (bit error rate) are obtained and recorded. The parameter used to implement and simulate the optical OR-gate is illustrated in Table 1.

As shown in Table 1, the wavelength for both data $A$ and data $B$ is $1556 \mathrm{~nm}$. Input data $A$ and data B are shown in Fig. 4 (a) and Fig. 4 (b), respectively. The injection current chosen to achieve the nonlinearity of the SOA at $250 \mathrm{Gbps}$ bit rate is 0.08 A. The waveform of the output of the optical logic 


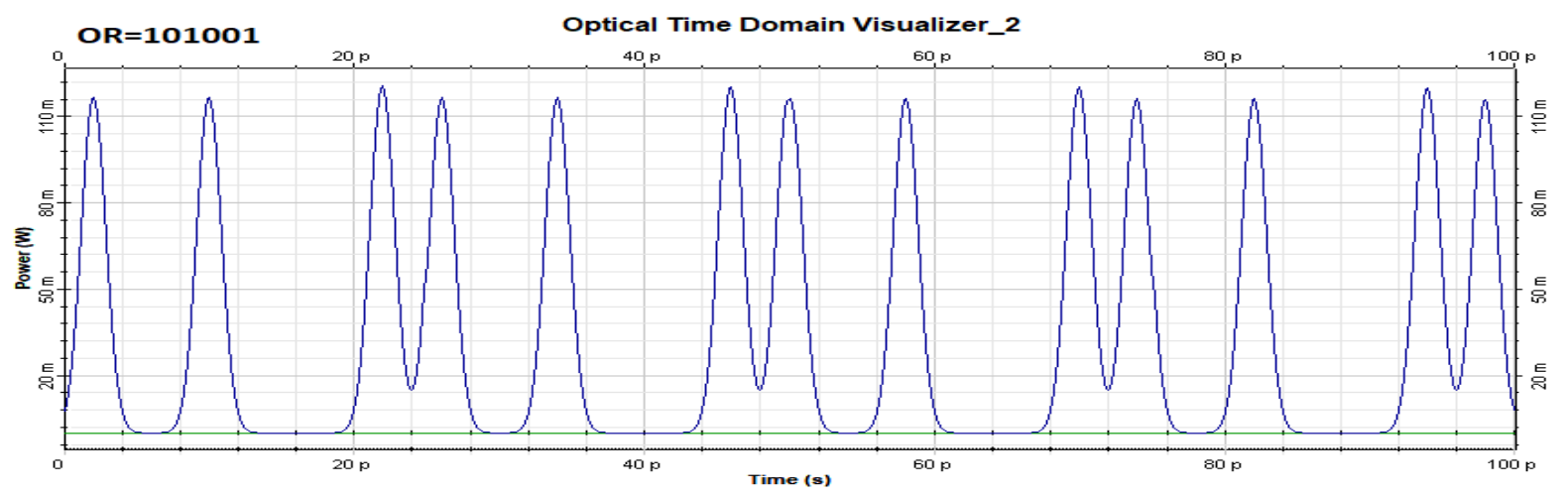

(a)

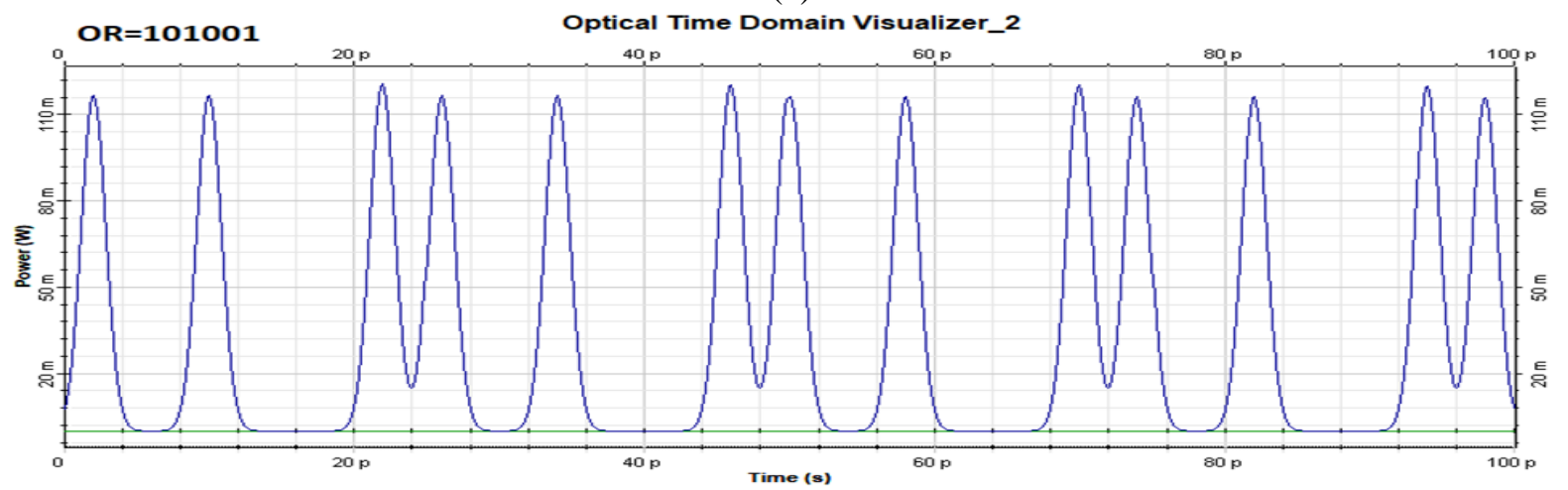

(b)

Figure. 5 The output waveform resulted from the OR gate and measured by optical time domain visualizer: (a) Before NRZ at 250Gbps, (b) after NRZ at 250Gbps

Table 1 The parameter used to implement the OR gate at $250 \mathrm{Gbps}$

\begin{tabular}{|c|c|c|c|}
\hline NO & The details & Value & unit \\
\hline 1 & Bit sequence generator $\mathrm{A}$ & 001001 & - \\
\hline 2 & Bit sequence generator $\mathrm{B}$ & 100000 & - \\
\hline 3 & $\begin{array}{l}\text { Optical Gaussian generator } \\
\text { Wavelength } \\
\text { Power }\end{array}$ & $\begin{array}{c}1556 \\
0.3\end{array}$ & $\begin{array}{l}\mathrm{nm} \\
\mathrm{mw}\end{array}$ \\
\hline 4 & $\begin{array}{l}\text { CW laser } \\
\text { Frequency } \\
\text { Power }\end{array}$ & $\begin{array}{l}- \\
-\end{array}$ & $\begin{array}{l}\mathrm{nm} \\
\mathrm{mw}\end{array}$ \\
\hline 5 & Injection current I & 0.08 & $\mathrm{~A}$ \\
\hline 6 & Bit rate & 250 & Gbps \\
\hline
\end{tabular}

gate OR before and after NRZ is shown in Fig. 5 (a) and Fig. 5 (b). The waveform of the output of the optical gate OR after low pass filter is shown in Fig. 6. The low pass filter is employed in this implementation to enhance the output waveform resulted from the NRZ.

The correct decision on whether the bit-type is " 1 " or " 0 " depends on the opening of the larger eye, which is calculated using Eye Diagram Analyzer. The larger the opening eye is, the greater the distinction among the levels of normal values of the signal for " 1 " and " 0 ". The greater that distinction is, the greater the Q-factor and the BER execution are. The eye diagram of the OR gate is shown in Fig. 7. It is shown from the eye diagram that the BER accomplished, and the max. Q-factor achieved at a bit rate of 250Gbps were 0 and 60.7989 , respectively.

By comparing the results obtained by the proposed design with those reported by [22, 23], we found that the results of the proposed design are much better than those reported by [22, 23]. The reason behind that was the parameters used to implement the optical OR gate, which were the wavelength of 1550 $\mathrm{nm}$, the injunction current of $230 \mathrm{~mA}$, sampling rates of $640 \times 10^{9} \mathrm{~Hz}$, and others. All these parameters and others are affecting the performance of the optical logic gates. Therefore, some adjustments were applied to the system designed by $[22,23]$ for the consideration of the fare comparison. Hence, both designs were implemented. Both designs were evaluated by different values of the injunction currents until the optimum value of the injunction current of each design was obtained. The optimum value of the injunction current means that the system design accomplishes high Q-factor with low BER.

Table 2 shows samples of the experimental results compared with those obtained by other stateof-the-art designs. The optimum value of the injunction current led to achieve the highest 


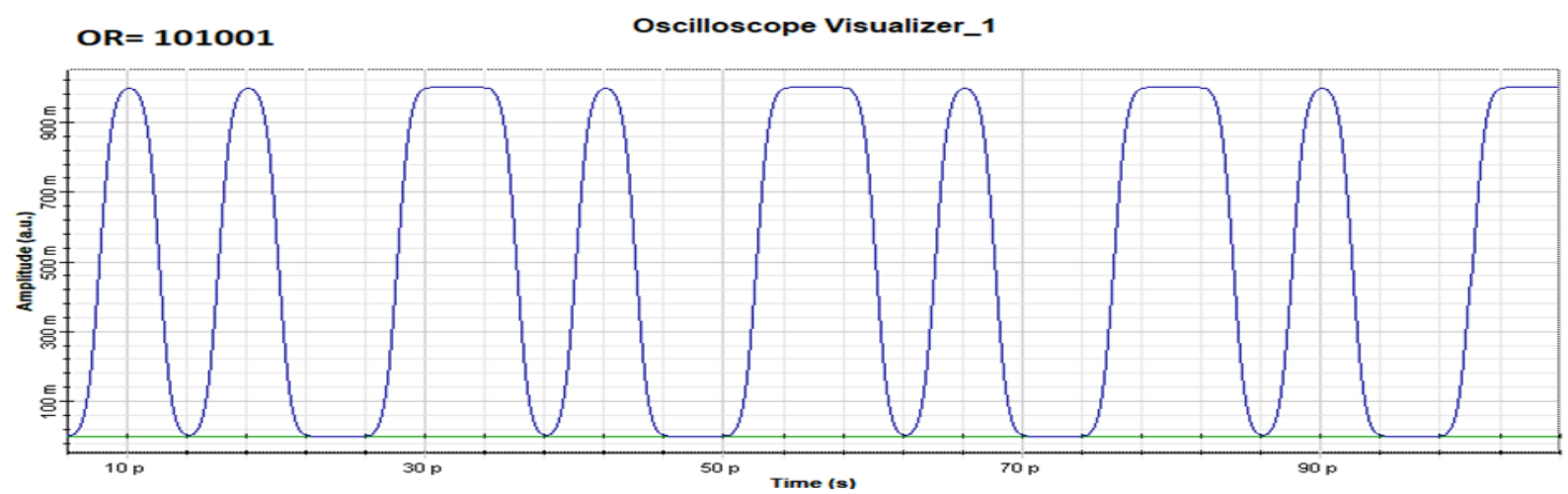

Figure. 6. The output of the OR gate from oscilloscope visualizer after the low pass filter at 250Gbps

Table 2. Samples of the simulation results of the OR gate at 250Gbps

\begin{tabular}{|c|c|c|c|c|c|c|}
\hline \multirow{2}{*}{$\begin{array}{c}\text { Injunction } \\
\text { current I }\end{array}$} & \multicolumn{2}{|c|}{ Proposed design } & \multicolumn{2}{c|}{ Design proposed by [22] } & \multicolumn{2}{c|}{ Design proposed by [23] } \\
\cline { 2 - 6 } & Q-factor & BER & Q-factor & BER & Q-factor & BER \\
\hline 0.065 & 20.213 & $2.04 \times 10^{-91}$ & 21.3 & $2.04 \times 10^{-65}$ & 18.93 & $1.73 \times 10^{-80}$ \\
\hline 0.07 & 32.022 & $1.43 \times 10^{-225}$ & 26.027 & $1.43 \times 10^{-76}$ & 27.724 & $9.22 \times 10^{-170}$ \\
\hline 0.075 & 50.638 & 0 & 27.325 & $1.43 \times 10^{-84}$ & 36.6366 & $1.95 \times 10^{-260}$ \\
\hline 0.08 & 60.7989 & 0 & 25.54 & $1.43 \times 10^{-91}$ & 37.61 & $3.83 \times 10^{-275}$ \\
\hline 0.085 & 49.766 & 0 & 22.911 & $1.43 \times 10^{-98}$ & 32.52 & $1.38 \times 10^{-255}$ \\
\hline 0.09 & 38.703 & 0 & 20.52 & $1.43 \times 10^{-101}$ & 27.433 & $2.84 \times 10^{-166}$ \\
\hline 0.095 & 31.736 & $1.28 \times 10^{-221}$ & 18.557 & $1.28 \times 10^{-104}$ & 23.674 & $1.72 \times 10^{-124}$ \\
\hline 0.1 & 27.2995 & $1.11 \times 10^{-164}$ & 16.99 & $1.11 \times 10^{-82}$ & 20.98 & $2.66 \times 10^{-98}$ \\
\hline 0.105 & 24.297 & $5.45 \times 10^{-131}$ & 15.79 & $5.45 \times 10^{-75}$ & 19.0414 & $2.04 \times 10^{-81}$ \\
\hline 0.11 & 22.148 & $2.78 \times 10^{-109}$ & 14.67 & $2.78 \times 10^{-63}$ & 17.566 & $1.17 \times 10^{-68}$ \\
\hline
\end{tabular}
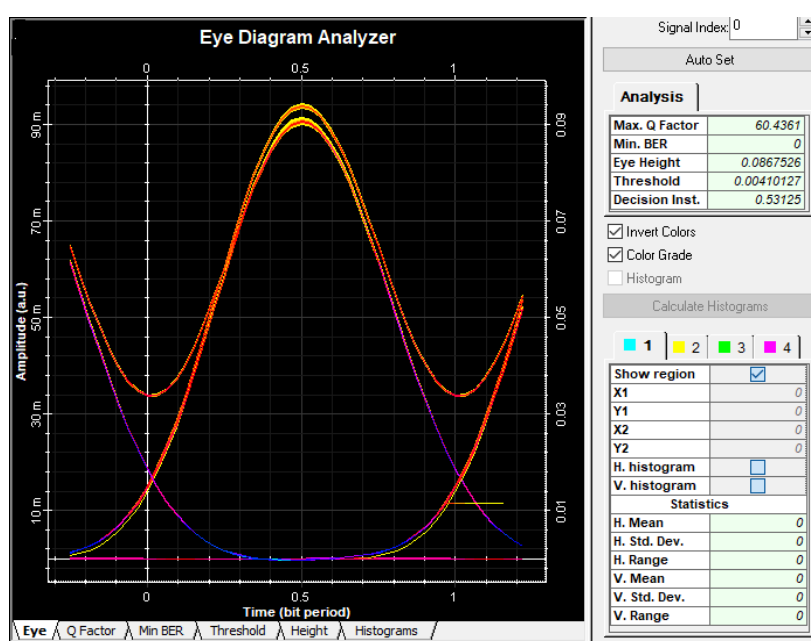

Figure. 7 Shows the eye diagram for the optical OR gate at $250 \mathrm{Gbps}$

performance in terms of Q-factor, BER, power consumption, etc. compared to the performance achieved by other designs. After implementing and simulating the optical OR gate that was presented by A.H. Neama and I. S. Desher [22] in 2017, we found the optimum injunction current, which was 0.075 . This injunction current led to obtain the Q-factor of 27.325 and BER of $1.43 \times 10^{-83}$. Therefore, the design proposed showed an excellent performance

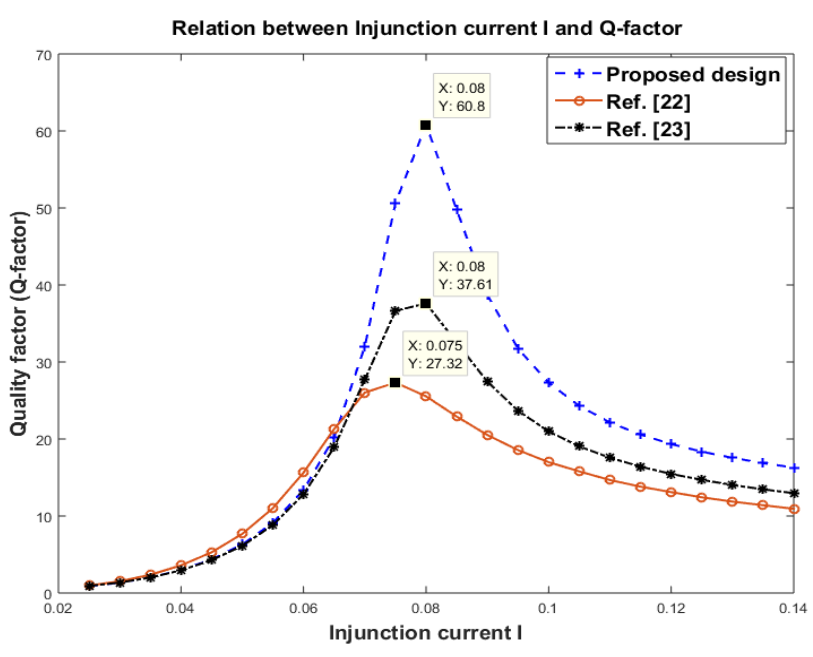

Figure. 8 Shows the relation between the injunction current I and the Q-factor

compared to the performance resulted in [22]. The design proposed showed the same performance when compared to the design proposed by A. Kotb and C. Guo [23] in 2020. The optimum injunction current, which is $0.08 \mathrm{~A}$, led to accomplish the BER and the Q-factor of $3.83 \times 10^{-275}$ and 37.61 , respectively.

Figs. 8 and 9 are shown the relation between different values of the injunction currents and the Q- 
Realtion between Injunction current I and BER

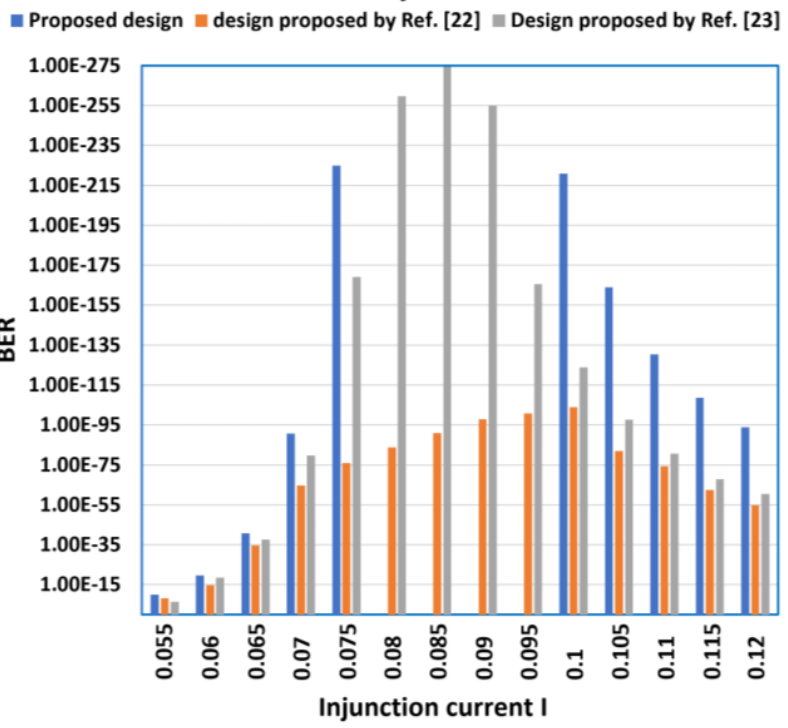

Figure. 9 Shows the relation between the injunction current I and the BER

factor and the BER, respectively. As shown in Fig. 8, when $\mathrm{I}=0.08 \mathrm{~A}$, the proposed design achieved highest Q-factor compared with those obtained in [22, 23]. Also, it is clearly noticeable in Fig. 9 that the values of BER obtained by the design proposed, which are 0 as shown in Table 2, are not appeared in Fig. 9 since the axes was logarithm base 10. In both Figs. 8 and 9, the experimental results of the design proposed is shown the excellent performance compared to those results obtained by $[22,23]$. This is due to the optimum designing parameters chosen to implement the circuit proposed of the OR gate and the parameters used to implement the SOA.

\section{Conclusion}

The design based on using MZI as a switch and employing SOA with the cross-gain modulation (XGM) to implement and simulate the optical logic gate OR at a high bit rate of $250 \mathrm{Gbps}$ was proposed in this paper. The optical logic gate OR was successfully implemented and simulated in this paper at $250 \mathrm{Gbps}$. The nonlinearity property of the SOA was employed in this paper to achieve the switching feature. The optimum value of the injection current, $\mathrm{I}=0.08 \mathrm{~A}$, was obtained to achieve the smallest value of the BER and the largest value of the Quality factor (Q), which are 0 and 60.7989, respectively. Hence, the SOA was biased using the optimum injection current. This led to decrease overall power consumption. The low pass filter was employed to enhance the output waveform resulted from the NRZ. The results of the design proposed in terms of the Qfactor and BER, shown in Table 2, outperformed those results obtained by $[22,23]$. The simulation was done by using the OptiWave software 17.

\section{Conflicts of interest (Mandatory)}

"The authors declare no conflict of interest."

\section{Author contributions (Mandatory)}

"Conceptualization, Ahmed Aldhahab; methodology, Ahmed Aldhahab; software, Ahmed Aldhahab; validation, Ahmed Aldhahab; formal analysis, Ahmed Aldhahab, Muhnaad Al-hayali, and Ibrahim Murdas; investigation, Ahmed Aldhahab; resources, Ahmed Aldhahab; data curation, Ahmed Aldhahab, Muhnaad Al-hayali, and Ibrahim Murdas; writing - original draft preparation, Ahmed Aldhahab; writing - review and editing, Ahmed Aldhahab; visualization, Ahmed Aldhahab; supervision, Ahmed Aldhahab; project administration, Ahmed Aldhahab; funding acquisition, Ahmed Aldhahab", etc. Authorship must be limited to those who have contributed substantially to the work reported.

\section{References}

[1] R. S. Tucker, G. Eisenstein, and S. K. Korotky, "Optical Time-Division Multiplexing for Very High Bit-Rate Transmission", Journal of Lightwave Technology, Vol. 6, No. 11, pp. 17371749, 1988.

[2] S. Singh and N. Singh, "Nonlinear effects in optical fibers: Origin, management and applications", Progress Electromagnetics Research, Vol. 73, pp. 249-275, 2007.

[3] N. Pleros, G. T. Kanellos, C. Bintjas, A. Hatziefremidis, and H. Avramopoulos, "Optical Power Limiter Using a Saturated SOA-Based Interferometric Switch", IEEE Photonics Technology Letters, Vol. 16, No. 10, 2004.

[4] V. Sasikala and K. Chitra, "Effects of Cross Phase Modulation and Four Wave Mixing in DWDM Optical Systems Using RZ and NRZ Signal", Gnanagurunathan G., Sangeetha R., Kiran K. (eds) Optical and Microwave Technologies. Lecture Notes in Electrical Engineering, pp. 53-63, 2017.

[5] V. Sasikala and K. Chitra, "All Optical Switching and Associated Technologies: A Review", Journal of Optics, Vol. 47, No. 3, pp. 307-317, 2018.

[6] N. Nozhat, H. Alikomak, and M. Khodadadi, "All-Optical XOR and NAND Logic Gates Based on Plasmonic Nanoparticles", Journal of Optics Communications, Vol. 392, pp. 208-213, 2017. 
[7] J. M. Oliveira, F. B. D. Sousa, F. M. D. Sousa, J. E. D. Oliveira, M. B. C. Costa, and M. B. C. Costa, "A New System for All Optical AND Logic Gate on Semiconductor Optical Amplifier Based Michelson", In: Proc. of 3rd International Symposium on Instrumentation Systems, Circuits and Transducers (INSCIT), Bento, Brazil, pp. 1-6, 2018.

[8] M. S. Islam and M. J. Barsha, "Mach Zander Interferometer (MZI) as A Switch for All Optical Network", In: Proc. of International Conf. on Innovation in Engineering and Technology (ICIET), Dhaka, Bangladesh, pp. 15, 2018.

[9] E. M. E. Saeed, A. A. E. Aziz, H. A. Fayed, and M. H. Aly, "Optical Logic Gates Based on Semiconductor Optical Amplifier Mach-Zander Interferometer: Design and Simulation", Optical Engineering, Vol. 55, No. 2, pp. 025104 (1-12), 2016.

[10] V. Sasikala and K. Chitra, "Performance Analysis of Multilogic All-Optical Structure Based on Nonlinear Signal Processing in SOA", Journal of Optics, Vol. 49, pp. 208-215, 2020.

[11] F. D. Mahad, A. S. M. Supaâ, S. M. Idrus, and D. Forsyth, "Review of Semiconductor Optical Amplifier (SOA) Functionalities", Jurnal Teknologi, Vol. 55, No. 1, pp. 85-96, 2012.

[12] N. K. Dutta and Q. Wang, Semiconductor optical amplifiers, World scientific, 2013.

[13] G. P. Agrawal, Fiber-Optic Communication Systems $5^{\text {th }}$ Edition, John Wiley \& Sons, 2021.

[14] A. Alquliah, A. Kotb, S. C. Singh, and C. Guo, "All-Optical AND, NOR, And XNOR Logic Gates Using Semiconductor Optical AmplifiersBased Mach-Zehnder Interferometer Followed by A Delayed Interferometer", Optik, Vol. 225, pp. 165901 (1-13), 2021

[15] Kotb, "Simulation of High Quality Factor AllOptical Logic Gates Based on Quantum-Dot Semiconductor Optical Amplifier at $1 \mathrm{~Tb} / \mathrm{S}$ ", Optik, Vol. 127, No. 1, pp. 320-325, 2016.

[16] Y. Akashi, S. Matsui, S. Isawa, A. Matsushita, A. Matsumoto, Y. Matsushima, H. Ishikawa, and K. Utaka, "Demonstration of All-Optical Logic Gate Device Using MQW-SOA and 10 Gbps XNOR Operation”, Physica Status Solidi, Vol. 216, No. 1, p.1800529 (1-6) , 2019

[17] M. J. Li, S. Li, and D. A. Nolan, "Nonlinear Fibers for Signal Processing Using Optical Kerr Effects", Journal of Lightwave Technology, Vol. 23, No. 11, pp. 3606-3614, 2005.

[18] Li, Nonlinear Optics Principles and Applications, Shanghai Jiao Tong University
Press, Shanghai and Springer Nature Singapore, 2017

[19] M. Moreno, "Kerr effect", São Carlos, Brazil: Instituto de Física de São Carlos-Universidade de São Paulo, pp. 1-4, 2018.

[20] P. Singh, A. K. Singh, V. Arun, and H. K. Dixit, "Design and Analysis of All-Optical Half-Adder, Half-Subtractor and 4-Bit Decoder Based on SOA-MZI Configuration", Journal of Optical and Quantum Electronics, Vol. 48, pp. 159 (114), 2016.

[21]K. Singh, G. Kaur, M. L. Singh, "Performance Analysis of An All-Optical Half-Subtracter Based on XGM in SOA at 20 Gbps", Journal of Optoelectronics and Advanced Materials Rapid Communications, Vol. 11, No. 3-4, pp. 189-196, 2017.

[22] A. H. Neama and I. S. Desher, "High Data Rate Optical logic OR, and NOT Gates at Optimum Injection Current based on SOA-MZI", International Journal of Computer Applications, Vol. 1, No. 3, pp. 24-32, 2017.

[23] A. Kotb and C. Guo, "All-optical multifunctional AND, NOR, and XNOR logic gates using semiconductor optical amplifiers", Physica Scripta, Vol. 95, No. 8, pp. 1-7, 2020. 